\title{
UM SISTEMA NACIONAL DE AQUISIÇÃO E PROCESSAMENTO DE DADOS ACOPLADO "ON LINE" AO ESPECTRÓMETRO DE MASSA DE FONTE SÓLIDA TH-5 (VARIAN MAT)
}

\author{
K.Kawashita \\ K.Sato' \\ I.K.Sonokir
}

\section{RESUMO}

Um espectrômetro de massa de fonte sólida da VARIAN MAT, modelo TH-5, utilizado em aplicações geocronológicas, foi acoplado "on line" ao microcomputador HP-9825B de 16 bits, tendo um voltímetro digital e interfaces apropriadas da Hewlett-Packard. O protótipo desenvolvido permite, através da técnica de "peak switching" de 8 canais controlados por uma sonda de efeito Hall, medidas isotópicas de todos os elementos analisáveis pelo método de ionização térmica. 0 sistema utilizado desde 1983, com amplo sucesso, rivaliza com os sistemas modernos conforme medidas efetuadas em 2 carbonatos padrões de estrôncio NBS-987 e SrN (E \& A). Experiência semelhante, utilizando um microcomputador da linha Apple, periféricos e interface apropriados e inteiramente nacionais desenvolvidos para o sistema, reproduziu, de forma bastante adequada, a performance anterior. A interface desenvolvida, pela sua versatilidade, pode acionar periféricos e acessórios como válvulas solenóides, sistemas de vácuo, sistema de troca de escala, etc, operações comuns na espectrometria. O software, desenvolvido nas linguagens HPL e Applesoft, é provido de correção de interferência dinâmica, técnica de interpolação de Dodson, rejeição de razões espúrias e cálculos de todos os parâmetros estatísticos normais.

\footnotetext{
${ }^{1}$ Centro de Pesquisas Geocronológicas/CPGeo, Instituto de Geociências/USP, São Paulo.
} 


\section{ABSTRACT}

A VARIAN MAT solid source mass spectrometer, varian model MAT TH-5, used for geochronological applications has been coupled on line to a 16-bit HP-9825B computer. An appropriate digital voltimeter and interface, both supplied by Hewlett-Packard, complete the system. The developed prototype allows, through peak switching of 8 channels controlled by a Hall effect probe, isotopic analyses of all the elements which can be carried out by the thermal ionization method. This system has been used since 1983 with success, and the strontium measurements performed on 2 carbonate standards NBS-987 and SrN (E\&A) agree, within experimental error, with results obtained in modern instruments. A similar system, with the same performance capabilities as the one described above, using an Apple computer and peripherals available in Brazil, has also been developed. The developed interface is provided with outputs to control external devices and accessories like solenoid valves, vacuum systems, scales, etc, common is mass spectrometry. The software, available in HPL and Applesoft languages, is provided with dinamic interference correction, Dodson's interpolation technique, rejection of the spurious ratios and computes all the common statistical parameters.

\section{INTRODUÇÃO}

A medida rigorosa da abundância isotópica de elementos teve grande avanço a partir da década passada, com o acoplamento de computadores "on line" aos espectrômetros de massa. Como um exemplo pioneiro, podemos destacar o trabalho executado por WASSERBURG et al. (1969). Antes, as relações isotópicas eram medidas diretamente nos espectrogramas, mediante o uso de réguas comuns ou, quando muito, com os conhecidos "chart rules". Além das limitações decorrentes do sistema de medida, havia ainda outras como os erros devidos a fatores de escala, falta de lineariedade do registrador, etc.

Hoje, os computadores constituem praticamente um acessório obrigatório e o seu custo, em relação aos espectrômetros, tem sido cada vez menor. Além de propiciar melhor precisão nas relações isotópicas, os computadores podem ainda controlar uma série de dispositivos periféricos, automatizando e uniformizando várias tarefas antes feitas manualmente. Os progressos conseguidos, até hoje, no sistema de processamento de dados em espectrometria, são tais que se pode considerar totalmente superada a fase experimental. Por exemplo, existem em atividade espectrômetros de fonte sólida que podem abrigar até 16 amostras, que são analisadas 
sucessivamente, de forma automática, e sem qualquer interferência humana.

Para espectrômetros de massa da área de química inorgânica existem, basicamente, dois tipos de sistemas de medida que são: medidas de razões isotópicas utilizando coletor simples ou múltiplo e medidas de diferenças, em partes por mil $(\delta \%$ ),em razões isotópicas, por exemplo,de ${ }^{13} \mathrm{C} /{ }^{12} \mathrm{C},{ }^{18} \mathrm{O} /{ }^{16} \mathrm{O}, \mathrm{D} / \mathrm{H},{ }^{15} \mathrm{~N} /{ }^{14} \mathrm{~N} e^{34} \mathrm{~S} /{ }^{32} \mathrm{~S}$.

Nos instrumentos de coletor simples como VARIAN MAT TH-5, as razões isotópicas são obtidas, geralmente, utilizando a técnica de "peak switching", em que as posições dos diversos "picos" (isótopos) ou "bases" são estabelecidas através do computador, que geralmente aciona uma unidade seletora de massas. Nos espectrômetros de setor magnético, a seleção de massas é feita mediante a utilização de um campo magnético programável, no qual o efeito de histerese é contornado através de sonda de efeito Hall (ver Fig.1). Geralmente cerca de 10 a 15 canais ou posições de massa, que correspondem biunivocamente a voltagens de referência bem determinadas, são suficientes. Os canais são selecionados em seqüência pré-estabelecida e a corrente iônica a ser medida é, em geral, integrada por um voltímetro digital integrador (DVM).

Nos espectrômetros providos de imã permanente, o sistema seletor de massas é mais simples, já que não há correções do tipo histerese. O "peak switcher", neste caso, fornece apenas as tensões de aceleração distintas para cada canal. Existem, no entanto, algumas restrições como a baixa resolução propiciada pelo valor de campo, relativamente limitado, dos imãs permanentes, ou os efeitos de discriminação de massas resultantes da variação na aceleração dos íons.

$\mathrm{Na}$ técnica de duplo coletor, o que se mede é a diferença na razão isotópica de um determinado elemento, relativa a um padrão do mesmo elemento. A amostra e o padrão são analisados diversas vezes e de forma intercalada, por meio de um sistema duplo de introdução. Essa medida é feita pelo método preconizado por McKINNEY et al. (1950), o método nulo, utilizando-se dois amplificadores independentes e um potenciômetro, de modo que uma fração do sinal amplificado, correspondente aos íns mais abundantes, anule o sinal produzido pelos íons menos abundantes.

Neste sistema de medição a precisão é alta, da ordem de $0,02 \%$. Mesmo assim, os espectrômetros modernos com duplo coletor podem também ser acoplados a um sistema de aquisição de dados, visto que o computador pode comandar toda uma série de tarefas, tais como:

1) checagem do sistema de vácuo do espectrômetro;

2) bombeamento do reservatório de amostra e do "manifold";

3) introdução da amostra e balanceamento das pressões;

4) limpeza do sistema após a análise, e admissão automática da amostra seguinte.

Neste trabalho abordaremos, com certo detalhe, um sistema nacional de aquisição 
e processamento automático de dados desenvolvidos no Centro de Pesquisas Geocronológicas do IG/USP, para espectrômetro de massa de fonte sólida, modelo TH-5 da VARIAN MAT, utilizado para análises isotópicas de $\mathrm{Sr}, \mathrm{Rb}$ e $\mathrm{K}$.

\section{SISTEMA NACIONAL E ADAPTAÇÃO AO ESPECTRÔMETRO DE MASSA TH-5}

O sistema nacional que passamos a descrever foi todo baseado em uma experiência adquirida anteriormente, utilizando elementos totalmente importados da Hewlett Packard (HP). Embora este sistema tenha sido aprovado plenamente, está sendo desativado por problemas ligados à manutenção do microcomputador HP-9825, que está fora de linha.

Assim, visando a nacionalização total do sistema de aquisição e processamento de dados, procurou-se como equivalente ao sistema HP, elementos mais comuns e disponiveis no mercado nacional. O sistema selecionado, cujas partes integrantes acham-se esquematizadas na Figura 1, consta dos seguintes elementos:

1) microcomputador da linha Apple de 8 bits com $64 \mathrm{~Kb}$ de memória;

2) impressora Elgin Lady;

3) voltímetro digital de $41 / 2$ dígitos com saída BCD;

4) interface IV para acoplamento do voltímetro digital ao microcomputador;

5) interface III de comando, via microcomputador, da fonte do eletroimã (corresponde ao "peak switcher") e troca automática de escala

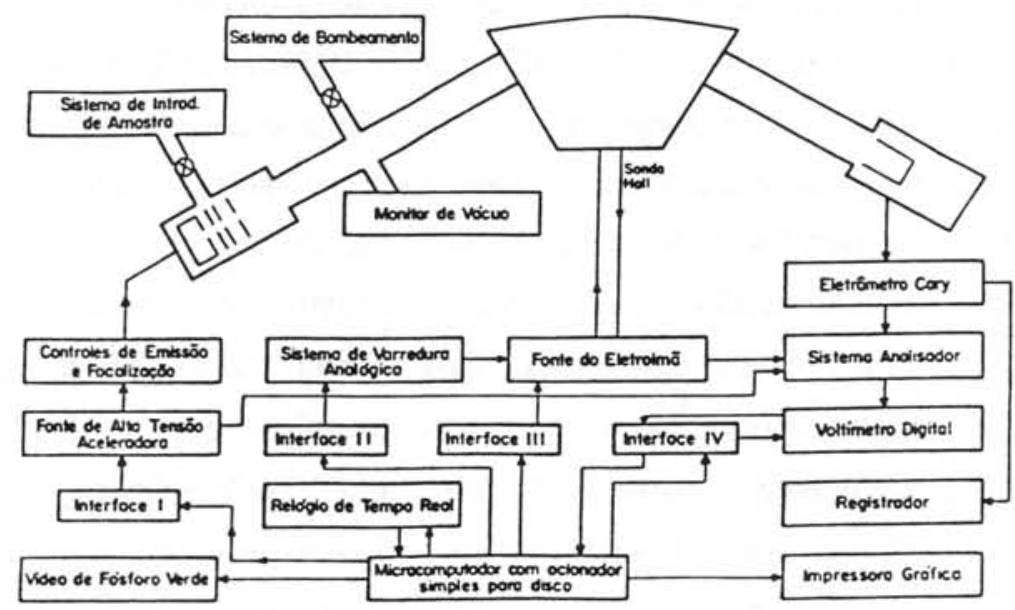

Figura 1 - Desenho esquemático do espectrômetro de massa computadorizado. 
As interfaces I e ll e o relógio de tempo real foram desenvolvidos para o sistema de informatização, empregado para o espectrômetro de fonte gasosa da marca Nuclide, e que não constitue objeto deste trabalho.

O voltímetro digital integrador escolhido foi o DVM modelo ND-045D de 4 1/2 dígitos, com opção BCD, de Equipamentos Cientfficos do Brasil, com integração de 1 segundo, préajustada pelo fabricante.

O microcomputador escolhido foi o Microengenho lle da Spectrum, da conhecida linha Apple, que se revelou plenamente adequado para as nossas finalidades. A única deficiência foi referente à fonte estabilizadora, que se mostrou inadequada e insuficiente, quando da inserção de novas interfaces que foram desenvolvidas especialmente para o projeto. Uma fonte estabilizadora com cerca do dobro da capacidade original revelou-se adequada.

Em relação ao projeto com a HP, algumas pequenas mudanças tiveram que ser efetuadas. Conforme aparece ilustrado na Figura 2, o DVM (ECB) nacional utilizado, por ter um alcance de apenas $200 \mathrm{mV}$, não pode ser acoplado diretamente à saída do eletrômetro existente. $O$ mesmo foi acoplado em paralelo com o registrador de papel. Os sinais maiores ou iguais a $200 \mathrm{mV}$, nesta saída, são atenuados automaticamente, via computador, através do acionamento das chaves K2, $\mathrm{K} 4, \ldots$, que correspondem a um determinado pico (canal).

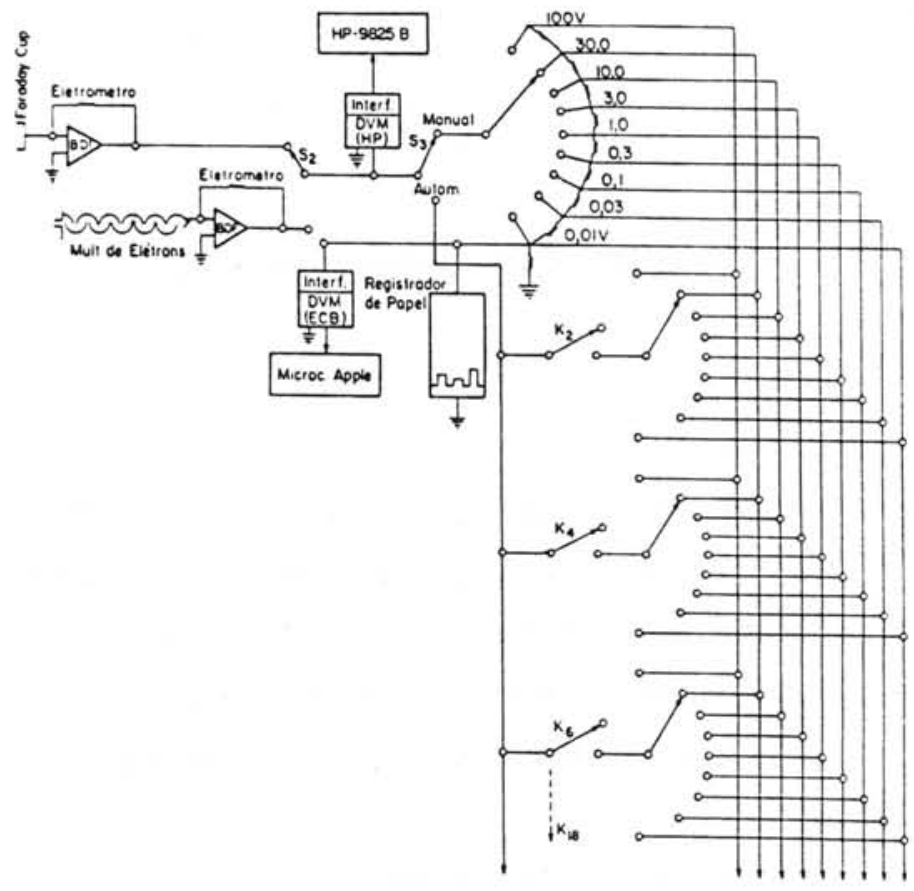

Figura 2 - Sistema detetor e seletor de escalas. 
A estratégia adotada, quando comparada com o sistema HP, tem um grande inconveniente em decorrência das resistências divisoras de tensão; cuja precisão é, em geral, da ordem de $0,1 \%$. Como as razões isotópicas devem ser medidas com precisão melhor, pelo menos 0,05\%, tivemos o cuidado de aferir a precisão destas resistências, utilizando pontes divisórias de tensão da General Radio, fontes super estabilizadas e DVM acoplado "on line" ao microcomputador Apple. Os fatores de calibração ou correção de escala obtidos foram, com exceção em uma das escalas, menores do que $0,1 \%$.

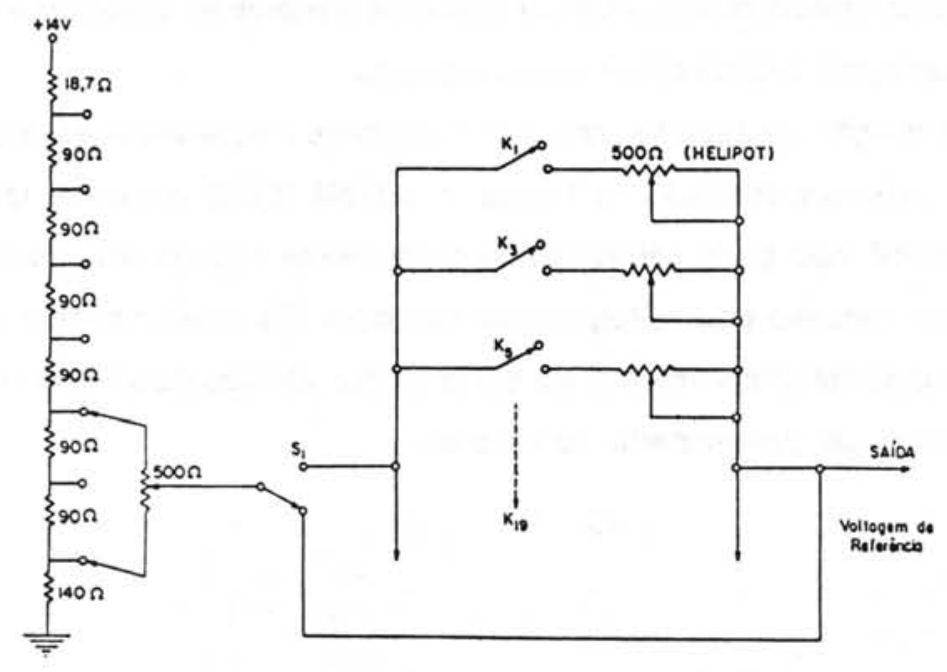

Figura 3 - Sistema seletor de massas.

O sistema seletor de massas (Fig. 3) bem como o seu respectivo comando (Fig. 4) são, praticamente, os mesmos daqueles utilizados com o sistema HP. Conforme está ilustrado na Figura 5, apenas mudam os comandos responsáveis pelo ritmo ("clock") de mudança dos canais e "reset", que são devidas a linguagens distintas. A instrução POKE é bastante familiar aos usuários da linguagem BASIC e corresponde à instrução wtb em linguagem HPL, especffica dos microcomputadores HP-9825.

Adicionalmente, este sistema é dotado de um conversor $D / A$, o que permite uma varredura completa e estende, por isso, a sua aplicabilidade para outros campos da espectrometria. 


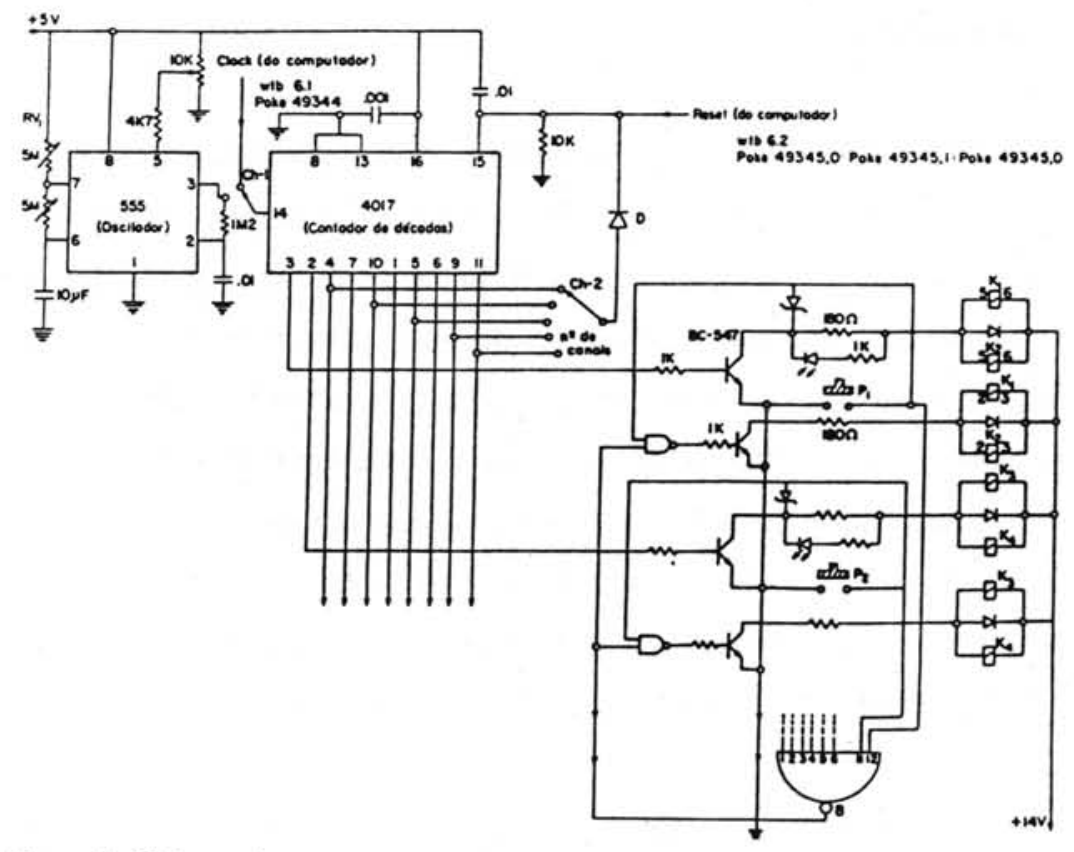

Figura 4 - Sistema de comando de seletor de massas.

Figura 5 - Interface de aquisição de dados e controle

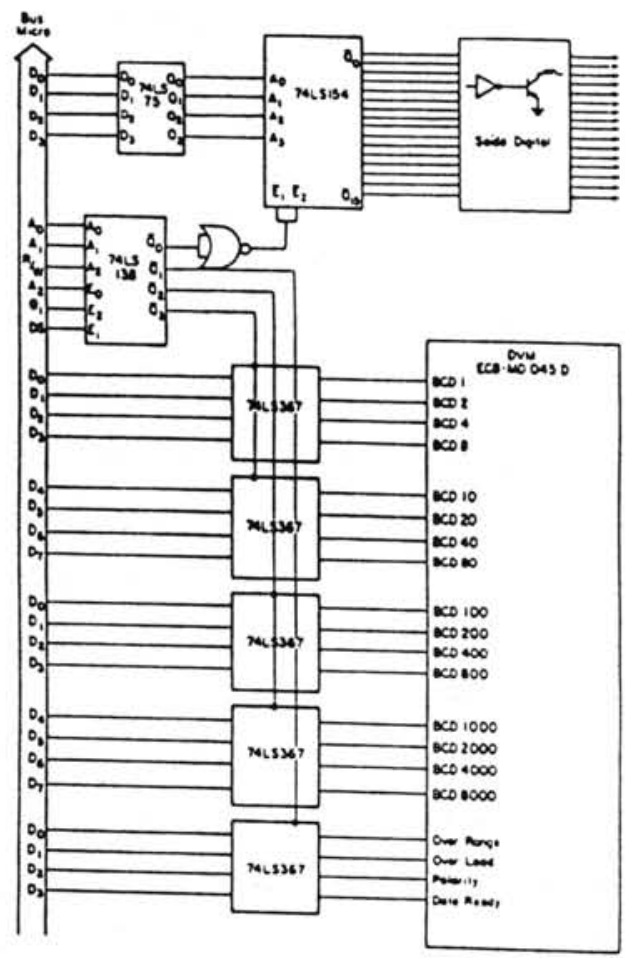




\section{AQUISIÇÃO DE DADOS E PROCESSAMENTO}

A aquisição de dados ou a obtenção de intensidade iônica em espectrometria de massa pode ser efetuada de modo analógico ou digital. No modo analógico, o campo magnético é variado linearmente ou não, de forma automática ou manual, e os diversos picos são registrados em um papel registrador. Após repetidos ciclos, em que as intensidades são, em geral, interpoladas, obtém-se razões isotópicas cuja precisão média é da ordem de $0,2 \%$. Tal procedimento, além de ser lento e ineficiente, é limitado quanto à precisão. A utilização de técnica digital e acoplamento "on line", controlando automaticamente o campo magnético permitiu como resultado principal, um considerável avanço na precisão e acurácia. Particularmente na geocronologia, novos métodos, que antes eram cogitados apenas teoricamente, passaram a ser utilizáveis rotineiramente, e nas outras metodologias estendeu-se à faixa de utilização em termos de idade, bem como melhorou-se a precisão das idades radiométricas obtidas.

Uma das interfaces desenvolvidas aparece na Figura 5. A mesma serve não só como "hardware" intermediador, entre o DVM e o computador, mas também para acionar ou comandar, através do computador, os dispositivos externos acopláveis, diretamente ou não, ao demultiplexador 74LS154. O protótipo montado permite acionar até 16 cargas ou comandos, o que julgamos mais do que suficiente na área de espectrometria inorgânica. Por exemplo, no acoplamento "on line" ao espectrômetro conforme detalhado na Figura 4, são necessários apenas 2 comandos (que foram discutidos anteriormente).

Foram desenvolvidos, na linguagem Applesoft, 3 "softwares" aplicativos que propiciam a aquisição automática e processamento de dados, quando da análise dos seguintes elementos:

1 - Sr natural: Picos $85,86,87$, Base e 88

2 - Sr com traçador: Picos $85,86,87$, Base, 88 e 84

3a - Rb com ou sem traçador: Picos 85,87 , e Base

3b - K com ou sem traçador: Picos Base, 39 e 41

O diagrama ilustrado na Figura 6 indica a seqüência de operações executadas pelo computador para a análise.

A aquisição e posterior tratamento de dados só é iniciada após a corrente iônica mais intensa do elemento alcançar, em geral, um valor da ordem de $10^{-11} \mathrm{~A}$, que é obtido manualmente. Alcançada esta corrente e selecionado o tipo de análise (1, 2, 3a ou 3b), que é função do tipo de elemento, o computador é acionado para executar o "jumping" (5 no caso do $\mathrm{Sr}$ natural), 


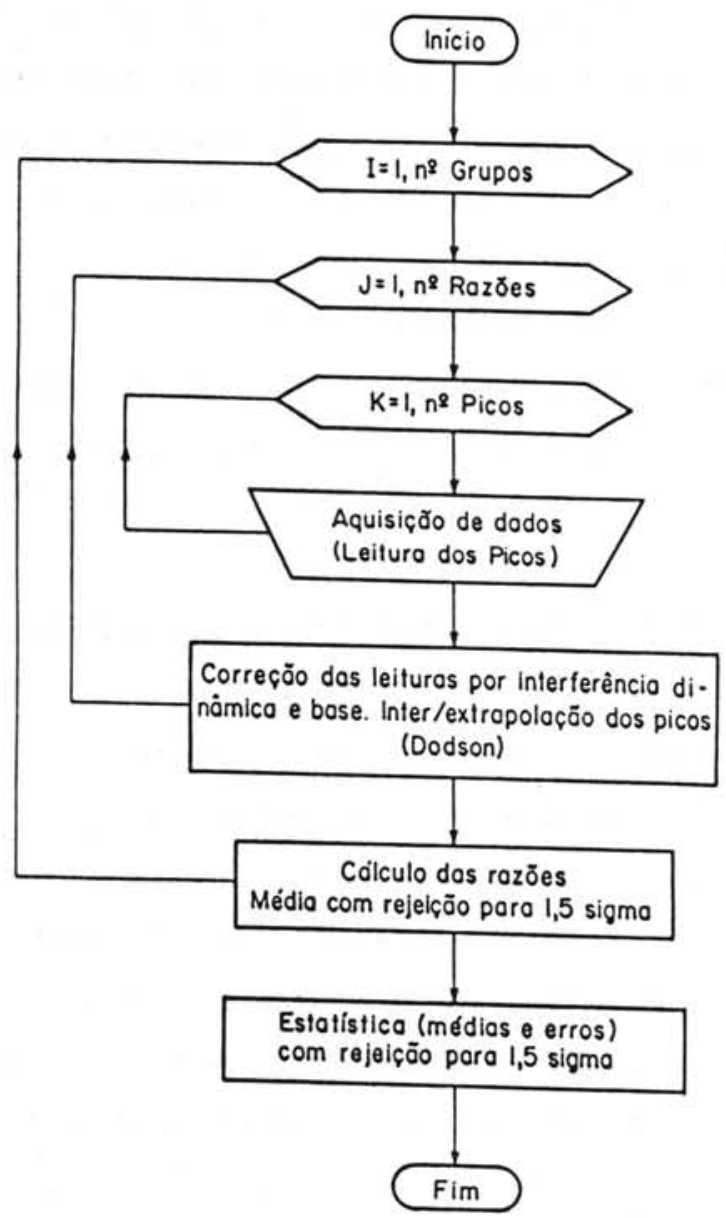

Figura 6 - Diagrama geral do software aplicativo para o espectrômetro TH-5.

permanecendo cerca de 6 segundos em cada canal (pico) pré-sintonizado, quando são efetuadas 3 integrações sucessivas de 1 segundo do respectivo sinal, após cerca de 3 segundos de espera. Este procedimento é efetuado $n$ vezes ( $n$ é difinido antes de cada grupo), propiciando, portanto, $n$ razões isotópicas. As intensidades são corrigidas para efeitos de interferência dinâmica (80 ppm) e a interpolação/extrapolação dos picos é efetuada segundo uma técnica apropriada para intensidades que variam de forma exponencial (técnica preconizada por M.H.Dodson da Universidade de Leeds, Inglaterra; P.N.Taylor, informação verbal).

As razões calculadas são submetidas a critérios de rejeição $(1,5 \sigma$, no nosso caso) e são calculados os erros estatísticos convencionais.

Normalmente são obtidos 6 ou 8 blocos (grupos), e as intensidades ou refocalizações podem ser ajustadas entre um grupo e outro. 
Em síntese, o "software" geral é constituído basicamente de 4 partes (sub-rotinas):

1 - aquisição e interpretação automática da seqüência de dados;

2 - cálculo das razões isotópicas mediante interpolação e extrapolação;

3 - tratamento estatístico do grupo de dados para um determinado número de ciclos, o que é definido momentos antes da aquisição de dados;

\section{4 - tratamento estatístico final.}

O relógio de tempo real, embora disponivel no sistema, é dispensado no "software" desenvolvido, uma vez que a aquisição de dados segue uma seqüência uniforme e constante.

\section{DESEMPENHO DO SISTEMA NACIONAL NO ESPECTRÓMETRO TH-5}

Medidas isotópicas precisas dependem não só do sistema particular de medida utilizado, mas também de uma série de fatores como: forma do topo do pico, estabilidade da corrente iônica, sistema de controle do campo magnético, voltagem de aceleração, etc.

Todos esses parâmetros, essenciais para a boa performance das medidas, podem ser facilmente levantados mediante programas especiais e com o "hardware" descrito. As características de desempenho de um espectrômetro de massa e do sistema de medidas empregado, podem ser melhor avaliadas analisando-se amostras padrão ou de referência bastante conhecidas: os carbonatos de estrôncio "Eimer and Amend" - SrN (E\&A) e NBS-987 do National Bureau of Standards.

Nas Tabelas 1 e 2, aparecem listados as razões ${ }^{87} \mathrm{Sr} /{ }^{86} \mathrm{Sr}$ normalizadas obtidas no Centro de Pesquisas Geocronológicas (CPGeo-IG/USP), no espectrômetro TH-5 e por outros laboratórios conceituados. Com exceção dos 2 resultados obtidos no CPGeo, que são médias de várias análises com leituras manuais dos gráficos (analógica) e que aparecem para simples comparação, todos os demais foram obtidos com saída digital, a maioria com computador "on line".

Propositalmente foram incluídos, para efeito de comparação, resultados obtidos em diversas marcas de espectrômetro, diferentes configurações e sistemas de medições e número de análises distintas.

Com referência ao carbonato de estrôncio NBS-987, o resultado mais preciso é o apresentado por S.Hart. No entanto, seu valor de 0,71016 não concorda, dentro do critério de $2 \sigma$, com outros valores de precisão similar, como os obtidos pela MAT e VG, os mais conceituados fabricantes europeus de espectrômetros. "A priori", é difícil dizer se as discordâncias se prendem à diferença real existente na razão isotópica, por causa da inomogeneidade da amostra, ou se são produzidas pelos 
Tabela 1 - Carbonato de estrôncio: NBS-987

Analista e/ou Laboratório

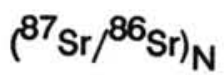

Observações

1. CPGeo/USP-C/H.P.

$0,71024 \pm 0,00006$

$(2 \sigma)$

$0,710235 \pm 0,000034 \quad(2 \sigma)$

2. MAT - Bremen

$0,710237 \pm 0,000032$

4. S. Hart (Inst. Carnegie)

$0,71016 \pm 0,00002$

5. C. Hedge - USGS

$0,71023 \pm 0,00007$

6. W. R.Shields - NBS

$0,71014 \pm 0,00020$

7. INGEIS - Argentina

$0,71033 \pm 0,00023$

8. NCGG - Pará

$0,71029 \pm 0,00006$

$(2 \sigma)$

Média (1 a 8)

$$
0,71023
$$

9. CPGeo-USP (analógico)

$0,71028 \pm 0,00036$

10. GPGeo-USP c/Apple

6 análises distintas

5 análises distintas

11 análises distintas

11 grupos (1 análise)

Certif. de análise

Certif. de análise

Certif. de análise

1 análise

1 análise

5 análises distintas

Tabela 2 - Carbonato de estrôncio: $\operatorname{Sr}_{N}(E \& A)$

Analista e/ou Laboratório

$$
\left({ }^{87} \mathrm{Sr} /{ }^{86} \mathrm{Sr}\right)_{\mathrm{N}}
$$

Observações

1. CPGeo-USP c/H.P.

$0,70800 \pm 0,00006$

$(2 \sigma)$

$0,707996 \pm 0,000027$

3. Univ. Oxford (Ingl.)

$0,70815 \pm 0,00005$

4. USGS - USA

$0,70797 \pm 0,00024$

5. INGEIS- Argentina

$0,70804 \pm 0,00024$

Média (1 a 5)

$$
0,70803
$$

6. CPGeo-USP (analógico)

$0,7081 \pm 0,0004$

$0,70795 \pm 0,00020$

4 análises distintas

9 análises distintas

8 análises distintas

16 análises distintas

1 análise

9 análises distintas

3 análises distintas 
sistemas de detecção utilizados. Se excluirmos os resultados de Hart, parece-nos que o melhor valor para o padrão NBS-987 deve ficar entre 0,7102 e 0,7103. O resultado médio obtido, com o sistema nacional (Apple), de 0,71023, é quase coincidente com o obtido com o sistema HP (importado) que $\theta$ de 0,71024 , ambos caindo, portanto, dentro desta falxa.

Para o carbonato de estrôncio "Eimer and Amend", um outro padrão interlaboratório, cedido ao CPGeo pelo Dr. S.Hart (lote \# 492327), foi compilado número menor de resultados (Tabela 2 e Fig. 7), já que, por ser tratar de uma amostra de referência relativamente antiga, poucos laboratórios modernos a possuem. Os dois resultados mais precisos, de Mainz e Oxford, não concordam entre si, e novamente é difícil determinar as causas possíveis da discrepância.

O resultado obtido com o sistema nacional (Apple), para este sal, mostra-nos um

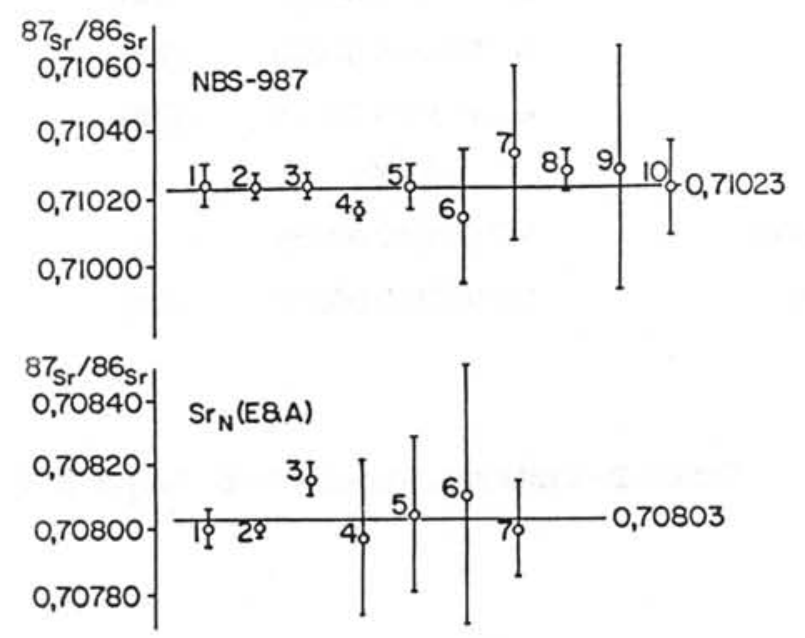

Figura 7 - Razões ${ }^{87} \mathrm{Sr} /{ }^{86} \mathrm{Sr}$ normalizadas nos carbonatos. Valores interlaboratórios de NBS-987 e SrN $(E \&$ A).

valor que é bastante concordante dentro do erro experimental, com o obtido pelo HP que é de 0,70800 , valor que melhor representa a razão ${ }^{87} \mathrm{Sr} /{ }^{86} \mathrm{Sr}$ normalizada.

Com base nestes resultados, podemos considerar o desempenho do sistema nacional como plenamente aceitável, sendo aconselhável incentivar todos os laboratórios, possuidores de espectrômetros $\mathrm{TH}-5$, a reproduzir a presente experiência.

Para medidas mais precisas do que aqui ilustradas para o elemento estrôncio, como aquelas requeridas para neodímio ou lutécio, é necessário ao menos um DVM de $51 / 2$ dígitos com saída BCD, para o qual, infelizmente, não existe similar nacional. O problema só pode ser parcialmente contornado, aumentando-se o período de integração dos picos de intensidade menor. 


\section{CONSIDERAÇÕES FINAIS E AGRADECIMENTOS}

O sistema aqui descrito vem, no nosso entendimento, preencher parcialmente, um dos planos da antiga RENEM/PRONAQ, ou seja, o de interfaciamento de espectrômetro com computadores brasileiros.

No Brasil, a maioria dos espectrômetros de setor magnético não são providos de tais sofisticações ou, quando existentes, não estão funcionando adequadamente por diversos motivos como: obsoletismo, falta de manutenção e/ou verba, falta de pessoal devidamente capacitado para operar, etc.

A unidade base, ou seja, o microcomputador escolhido por nós fol a da linha Apple, uma das mais simples e perfeitamente adequada para a espectrometria inorgânica. Quanto ao DVM só lamentamos que ainda não exista um de 5 1/2 digitos nacional e cujas qualidades sejam iguais aos utilizados por nós.

No quadro deste projeto, foram desenvolvidas as interfaces necessárias que não se qualificam como especfficas. Qualquer instrumento de saída analógica, com atenuação adequada, se for o caso, pode se valer do nosso sistema. Do mesmo modo, pode-se comandar qualquer carga habitual conectada adequadamente aos "drivers" de saída. Assim pode-se abrir ou fechar válvulas magnéticas, ligar automaticamente sirenes, abrir ou fechar portões, etc. A interface IV é, pois, mais do que um controlador lógico programável. Sua aplicação transcende os objetivos da espectrometria, entrando na faixa dos sistemas simples de automação, até nos sistemas controláveis pelo computador, como os utilizados na indústria.

Os componentes empregados são bastante simples e portanto de fácil manutenção, livrando-nos de uma tormentosa questão. Um outro aspecto bastante importante e que devemos salientar, foi o desenvolvimento de inúmeros "softwares" aplicativos, alguns fugindo até do escopo inicial.

Esperamos que este benefício todo, conquanto ainda restrito, atinja a outros interessados. Um trabalho, ainda na fase preliminar, foi apresentado, por ocasião do I Encontro Latino de Espectrometria de Massas em março de 86, no Rio de Janeiro e também exposto, mais recentemente em São Paulo, durante o $1^{\circ}$ Seminário de Instrumentação.

Finalmente, cabe aqui consignar os agradecimentos ao GT de Instrumentação, especialmente na pessoa da Enga Lygia Donadio, que não só teve a oportunidade de acompanhar o progresso dos nossos trabalhos através de relatórios e de seminários, bem como, por sua iniciativa e, a nosso pedido, complementar adequadamente e no momento oportuno o nosso projeto. 


\section{REFERÊNCIAS BIBLOGRÁFICAS}

Mc KINNEY, C.; Mc CREA, J.M.; EPSTEIN, S.; AШEN, H.A.; UREY, H.C. (1950) Improvements in mass spectrometers for the measurements of small differences in isotope abundance ratios. Rev. Sci.Instr., $21(8): 724-30$.

WASSERBURG, G.J.; PAPANASTASSIOU, D.A.; NENOW, E.V.; DAUMAN, C.A. (1969) A programable magnetic field mass spectrometer with on-line data processing. Rev.Sci.Instr., 40(2):288-295. 